\title{
Review Article \\ Pregnancy after Bariatric Surgery: A Review
}

\author{
N. L. Hezelgrave and Eugene Oteng-Ntim \\ Maternal and Fetal Research Unit, Kings College London, St. Thomas' Hospital, Westminster Bridge Road, London SE1 7EH, UK \\ Correspondence should be addressed to N. L. Hezelgrave, natasha.hezelgrave@kcl.ac.uk
}

Received 1 September 2010; Accepted 17 May 2011

Academic Editor: Francesco Saverio Papadia

Copyright ( 2011 N. L. Hezelgrave and E. Oteng-Ntim. This is an open access article distributed under the Creative Commons Attribution License, which permits unrestricted use, distribution, and reproduction in any medium, provided the original work is properly cited.

\begin{abstract}
Maternal obesity is a major cause of obstetric morbidity and mortality. With surgical procedures to facilitate weight loss becoming more widely available and demanded and increasing number of women becoming pregnant after undergoing bariatric surgery, it is important and timely to consider the outcome of pregnancy following bariatric surgery. This paper aims to synthesize the current evidence regarding pregnancy outcomes after bariatric surgery. It concludes that bariatric surgery appears to have positive effects on fertility and reduces the risk of gestational diabetes and preeclampsia. Moreover, there appears to be a reduced incidence of fetal macrosomia post-bariatric procedure, although there remains uncertainty about the increased rates of small-for-gestational age and intrauterine growth restricted infants, as well as premature rupture of membranes in this group. A number of case reports highlight that pregnancy following bariatric surgery is not without complications and it must be managed as high risk by the multidisciplinary team.
\end{abstract}

\section{Introduction}

The epidemic of obesity in middle- and high-income countries has led to an increased prevalence of obese women of reproductive age, conveying enormous consequences for the health of both mother and child. Maternal obesity (defined as BMI $\geq 30$ at the first antenatal consultation) has become one of the most commonly occurring risk factors in obstetric practice [1], and the prevalence of obesity in pregnancy has risen dramatically in recent years. In the UK, 33\% of pregnant women are overweight or obese [2]. Indeed, the Centre for Maternal and Child Enquiries (CMACE) has selected obesity in pregnancy as its principal project for 20082011, with emphasis that in the UK, between 2000-2003 approximately $35 \%$ of the women who died in pregnancy, childbirth, or in the postpartum period were obese. Furthermore, approximately $30 \%$ of mothers who had a stillbirth or neonatal death were obese [3].

Obese women have an increased risk of stillbirth or intrauterine fetal death [4]. They are at greater risk of preterm labour, miscarriage and fetal chromosomal anomalies, as well as macrosomia. Obese women are more likely to suffer from thromboembolism, gestational diabetes, pregnancyinduced hypertension, and preeclampsia. They have a greater incidence of dysfunctional labour, caesarian section [5] and associated perioperative morbidity, as well as postpartum haemorrhage. Therefore, weight loss after surgery has the potential to confer enormous health benefit for mother and child.

The health burden of obesity is driving increasing numbers of people, including women of reproductive age, to seek long-term treatment to facilitate weight loss. However, few anti-obesity interventions have been found to be helpful. Comparing surgery with nonoperative means of weight loss, the National Institute for Health and Clinical Evidence concluded.

Surgery remains more effective than a nonsurgical approach for people who are obese (BMI $>38$ for women, $>34$ for men) in the longer term (measured up to 10 years after surgery) [6].

A number of gastric banding procedures, many now done laparoscopically, have been shown to produce dramatic weight loss, and many women become pregnant after such procedures. Does weight loss by these surgical means confer the assumed health benefits of weight reduction? What are the risks to pregnancies post-bariatric procedures? 
Increasing numbers of research papers have sought to quantify the benefits for maternal and child health in such pregnancies, as well as highlighting potential complications of pregnancy after these procedures. This paper summarises the current evidence regarding pregnancy after bariatric surgery to draw conclusions regarding maternal, fetal and infant risks and benefits, and to highlight areas in which further robust research is required before recommendations can be made.

\section{Fertility}

There is a vast amount of evidence supporting the negative effects of obesity on fertility and on IVF outcomes. Characterised by a hyperinsulinaemic and potentially hyperandrogenaemic state, obesity may lead to oligo/amennorhoea, often in association with polycystic ovarian syndrome. The relative risk of anovulatory infertility has been found to be as high as 3.1 in patients with BMI > 27 [7]. Weight reduction has been shown to readdress this hormonal imbalance and increase fecundity in obese and overweight women [8]. It is therefore postulated that bariatric surgery improves menstrual regularity and ovulation in anovulatory obese women, thus restoring fertility.

Assessing fertility by a proxy of normalization of menstrual cycles, as well as lessening of symptoms of PCOS, a number of studies have demonstrated resumption of ovulation after bariatric surgery and associated weight loss. In a retrospective patient survey-based study, Teitelman et al. found a preoperative anovulatory rate of approximately half of women undergoing bariatric surgery (largely Roux-en$\mathrm{Y}$ gastric bypass), with resumption of regular menstrual patterns in $71.4 \%$ and a positive correlation between degree of weight loss and likelihood of resolution of menstrual dysfunction [9]. Furthermore, a prospective study of 17 obese patients with PCOS found decreased hirsutism and blood levels of testosterone, androstenedione, dehydroepiandrosterone sulfate, as well as normalization of menstrual cycles in all women after bariatric surgery [10].

Less has been published exploring the effect of bariatric surgery on spontaneous and IVF treatment-related fertility rates, largely as most studies rely on retrospective case-control studies from women who were able to get pregnant and whose pre-conception fertility histories were available [11]. Nevertheless, a number of small studies comparing fertility rates before and after surgery do report improvements in fertility $[12-14]$.

\section{Pregnancy Related Medical Complications}

There is increasing evidence to suggest that weight loss after bariatric surgery may improve maternal and perinatal outcomes by reducing obesity-associated obstetric risk factors. Although there is a paucity of robust randomized control trials in this area, a number of recent case-control and cohort studies demonstrate that women who have had preconceptual bariatric surgical procedures may have lower rates of obesity-related pregnancy complications such as gestational diabetes and hypertensive disorders than either historical controls or women who had pregnancies before their bariatric procedures $[15,16]$.

In a retrospective cohort study of US insurance claims of 585 women who had undergone bariatric procedures, Bennett et al. found that women who had delivered after their bariatric procedure $(N=269)$ had substantially lower rates of preeclampsia and eclampsia (odds ratio $0.20,95 \%$ confidence interval 0.09 to 0.44 ), chronic hypertension complicating pregnancy $(0.39,0.20$ to 0.74$)$, and gestational hypertension $(0.16,0.07$ to 0.37$)$, even after adjustment for age, multiple pregnancy, surgical procedure, and preexisting diabetes [17]. Similarly, in a large retrospective study of all women between 1988-2006 who delivered after bariatric surgery in a tertiary unit in Israel, Weintraub et al. found a significant reduction in the rates of gestational diabetes mellitus $(17.3 \%$ versus $11.0 \% ; P=0.009)$ and hypertensive disorders in pregnancy $(23.6 \%$ versus $11.2 \% ; P=0.001$ ) after analyzing 301 deliveries preceding bariatric surgery and 507 following surgery [18]. Wittgrove et al., in a retrospective study of 36 post Roux-en-Y gastric bypass pregnancies, found decreased rates of gestational diabetes mellitus, hypertensive disorders, as well as fetal macrosomia in the postsurgery group [19].

Not all studies concur with these results. Patel et al. did not find a statistically significant difference in pregnancyinduced hypertension, preeclampsia, nor gestational diabetes mellitus between postlaparoscopic Roux-en-Y treated patients and obese and nonobese controls [20]. Moreover, higher rates of gestational diabetes and chronic hypertension have been reported in patients postbariatric surgery, although this association was shown to be nonsignificant after adjustment for confounding factors [21].

\section{Perinatal Outcome}

As described above, bariatric surgery and the weight loss associated therewith appears to decrease obesity-related maternal morbidity. It therefore follows that perinatal outcome, particularly those associated with disorders such as gestational diabetes and preeclampsia, would be improved following bariatric surgery and pre-pregnancy weight loss. However, there lacks good evidence to support improved perinatal outcomes following pregnancy postbariatric surgery, although there is little evidence to suggest adverse outcomes.

\section{Birth Weight}

Maternal obesity is known to have implications on birth weight, particularly an increased risk of macrosomia (birth weight $>4000 \mathrm{~g}$ ) [22]. A number of retrospective and prospective observational studies have sought to define the impact of bariatric surgery on birth weight.

Pregnancy following bariatric surgery has also been demonstrated to reduce fetal macrosomia (birth weight > $4000 \mathrm{~g}$ ). Patel et al. found a significant decrease in mean birthweight and the incidence of macrosomia after Rouxen-Y gastric bypass compared with severely obese patients, 
and similar to those of nonobese and obese patients [20]. Similarly, Weintraub et al. found a significant reduction in the incidence of macrosomia in women who delivered before bariatric surgery compared with those who delivered before (7.6\% versus $3.2 \% ; P=0.004)$ [18]. Importantly, when comparing women with previous bariatric surgery $(n=298)$ with all deliveries $(n=159210)$ irrespective of maternal weight, women who had previous bariatric surgery were more likely to have macrosomic babies (OR 2.1, 95\% CI: 1.43.0, $P<0.001)$ [21].

Although there seems to be evidence to demonstrate a reduced incidence of macrosomia after bariatric surgery, the study by Sheiner et al. demonstrated an apparently increased rate of intrauterine growth restriction with a history of bariatric surgery (5\% versus $2 \%$; $P<0.001)$, as well as premature rupture of membranes (OR 1.9, $P=0.001$ ), although this association was not persistent after multivariable analysis (OR 1.4, $P=0.063$ ) [21]. Patel et al. demonstrated that the incidence of SGA after RYGB was higher (11.5\%) compared with nonobese patients $(0.5 \%, P<$ $0.001)$, but not significantly different from obese $(2.6 \%)$ and severely obese patients (3.7\%) [21]. Although Ducarme et al. demonstrated the rates for low birth weight $(<10 \%$ centile $)$ were lower amongst postbariatric surgery patients compared with controls $(7.7 \%$ versus $10.6 \%)$ [23], neither Richards et al. [24] nor Dixon et al. [25] demonstrated significant differences in the incidence of SGA infants in post-bariatric surgery patients compared with controls.

A reduced risk of macrosomia is not supported by all studies. In a prospective study of 79 consecutive pregnancies following laparoscopic adjusted gastric banding in comparison with the same patient's penultimate pregnancies before surgery, Dixon et al. found that although the pregnancy maternal weight gain was lower in those who had undergone surgery, there was no significant difference in birth weight between the two groups [25].

Timing of pregnancy after bariatric surgery has not been demonstrated to affect birth weight. In a small retrospective review, Dao et al. demonstrated that of 34 patients who became pregnant after bariatric surgery between 2001-2004, there was no significant difference in birth weight or premature labour between the 21 patients who fell pregnant within 1 year after surgery and the 13 who became pregnant $>1$ year after the procedure [26].

\section{Premature Birth and Miscarriage}

From the literature, it seems that the rate of premature delivery does not seem to significantly differ in pregnancies after bariatric surgery, compared control groups matched for BMI $[20,24,25]$, nor with those prebariatric surgery $[14,19,25]$. Nevertheless, a very recent meta-analysis of 84 cohort and case control studies has suggested that although the overall risk of preterm birth is similar in overweight, obese women and women of normal weight, the risk of induced preterm birth was increased in overweight and obese women (relative risk $1.30,1.23-1.37$ ), with causes presumably related to increased medical complications in pregnancy necessitating early delivery. It is also found that the overall risk of spontaneous or induced preterm birth before 32 weeks completed gestation is increased after bariatric surgery [27].

While one small case series of 9 patients reports a miscarriage rate decline of $33.3 \%$ to $7.8 \%$ in patients following bariatric surgery, [28] with the presurgery pregnancies as the control group, there is a lack of robust evidence to demonstrate a significant effect of bariatric surgery on miscarriage rate. Whilst a higher miscarriage rate $(21.6 \%)$ was demonstrated in bariatric surgery preoperative patients compared with the general population, this was not reduced postoperatively $(26 \%)$ despite a reduction in BMI [29].

\section{Perinatal Death and Congenital Malformations}

Despite the above-described apparent increase in IUGR and premature rupture of membranes (albeit before multivariable analysis), Sheiner et al. did not find a significant difference in perinatal mortality between those with a history of bariatric surgery [21]. Comparing all pregnancies of patients with and without previous bariatric surgery $(N=159210)$, the perinatal mortality rate was not significantly different between the groups. However, a number of observational studies have highlighted the importance of further research into the potential increase in congenital malformations following bariatric surgery; a prospective cohort of 239 pregnancies after BPD reported two birth malformations, one infant dying from surgery for meconium obstruction and two deaths from "unknown causes" [30].

\section{Maternal Nutritional Deficiencies}

Concern rightly exists surrounding the theoretical potential for nutritional deficiencies for mother and infant after bariatric surgery, particularly malabsorptive procedures. Published outcomes of adverse nutritional deficiencies in women who have undergone bariatric surgery are rare, although case reports do exist detailing deficiencies in iron, B12, calcium, and vitamin $\mathrm{D}$ after malabsorptive procedures which have the potential to lead to fetal complications including neural tube defects, low birth weight, neonatal hypocalcaemia or rickets [31]. Eerdekens et al. report on 5 cases of severe neonatal intracranial bleeding resulting in three neonatal deaths and two severely disabled infants, in births following bariatric surgery, all possible related to vitamin K deficiency [32]. Whilst disturbances of coagulation as a result of vitamin $\mathrm{K}$ deficiency was proved only in one of the cases (maternal gastric outlet obstruction 2 years post gastric banding), it highlights that careful attention to maternal and fetal nutrition must be made a crucial element of care in such pregnancies.

\section{Surgical Complications}

An increase in intra-abdominal pressure, displacement of organs by a gravid uterus, and a predisposition to vomiting in pregnancy poses risk to the pregnant woman post 
bariatric surgery. A systematic review of 75 research papers by Maggard et al. described 20 reports of complications requiring surgical intervention during pregnancy following bariatric surgery including 14 bowel obstructions, 1 gastric ulcer, 4 band events and 1 staple line stricture, resulting in 3 maternal, and 5 neonatal deaths [11]. Such reports highlight the importance of treating a pregnant woman after bariatric surgery as high-risk pregnancy although these rates may not indeed be higher than in the normal population when reporting bias is accounted for.

\section{Conclusions}

The evidence presented above is strongly suggestive that patients who undergo bariatric surgery may have lower risk of maternal complications such as preeclampsia and gestational diabetes compared with obese controls or presurgery pregnancies. Furthermore, it seems that bariatric surgery may reduce the risk of fetal macrosomia, although the risk of growth restriction or low birth weight is not clear and risk of nutritional deficiencies appears rare. Isolated case reports detailing intestinal obstruction and other surgical emergencies during pregnancy in women who have had bariatric surgery need further quantification, although it is clear that pregnancy postbariatric surgery is not without risks. Clearly, careful attention must be paid to women who have had previous surgery to ensure adequate maternal and fetal nutrition, as well as the early recognition of potential complications.

Unfortunately, the level of evidence regarding maternal and fetal outcomes after bariatric surgery is limited to observational case control and cohort studies; no definitive studies comparing pregnancy outcomes between different bariatric operative techniques have been performed, nor do any randomized control trials exist to definitively characterize the reproductive potential of bariatric surgery, nor are they likely to be feasible. Further research is needed to clarify the benefits and risks of prepregnancy bariatric surgery to better equip the multidisciplinary team with informed advice and management of preconceptual, antenatal, delivery, and postnatal care for these women.

\section{References}

[1] CMACE/RCOG Joint Guideline: Management of Women with Obesity in Pregnancy. CMACE, 2010.

[2] N. Heslehurst, L. J. Ells, H. Simpson, A. Batterham, J. Wilkinson, and C. D. Summerbell, "Trends in maternal obesity incidence rates, demographic predictors, and health inequalities in 36821 women over a 15-year period," British Journal of Obstetrics and Gynaecology, vol. 114, no. 2, pp. 187-194, 2007.

[3] Perinatal Mortality, 2005. Confidential Enquiry into Maternal and Child Health. CEMACH, 2007.

[4] E. A. Nohr, B. H. Bech, M. J. Davies, M. Frydenberg, T. B. Henriksen, and J. Olsen, "Pre pregnancy obesity and fetal death: a study within the Danish national birth cohort," Obstetrics and Gynecology, vol. 106, pp. 250-259, 2005.

[5] S. Y. Chu, S. Y. Kim, C. H. Schmid et al., "Maternal obesity and risk of cesarean delivery: a meta-analysis," Obesity Reviews, vol. 8, no. 5, pp. 385-394, 2007.
[6] Clinical Guideline No.43. Obesity: guidance on the prevention, identification, assessment and management of overweight and obesity in adults and children. National Institute for Health and Clinical Excellence Section 5: 638, 2006.

[7] F. Grodstein, M. B. Goldman, and D. W. Cramer, "Body mass index and ovulatory infertility," Epidemiology, vol. 5, no. 2, pp. 247-250, 1994.

[8] S. M. Nelson and R. Fleming, "Obesity and reproduction: impact and interventions," Current Opinion in Obstetrics and Gynecology, vol. 19, no. 4, pp. 384-389, 2007.

[9] M. Teitelman, C. A. Grotegut, N. N. Williams, and J. D. Lewis, "The impact of bariatric surgery on menstrual patterns," Obesity Surgery, vol. 16, no. 11, pp. 1457-1463, 2006.

[10] H. F. Escobar-Morreale, J. I. Botella-Carretero, F. ÁlvarezBlasco, J. Sancho, and J. L. San Millán, "The polycystic ovary syndrome associated with morbid obesity may resolve after weight loss induced by bariatric surgery," Journal of Clinical Endocrinology and Metabolism, vol. 90, no. 12, pp. 6364-6369, 2005.

[11] M. A. Maggard, I. Yermilov, Z. Li et al., "Pregnancy and fertility following bariatric surgery: a systematic review," Journal of the American Medical Association, vol. 300, no. 19, pp. 2286-2296, 2008.

[12] B. Bilenka, I. Ben-Shlomo, C. Cozacov, C. H. Gold, and S. Zohar, "Fertility, miscarriage and pregnancy after vertical banded gastroplasty operation for morbid obesity," Acta $\mathrm{Ob}$ stetricia et Gynecologica Scandinavica, vol. 74, no. 1, pp. 42-44, 1995.

[13] M. Deitel, E. Stone, H. A. Kassam, E. J. Wilk, and D. J. A. Sutherland, "Gynecologic-obstetric changes after loss of massive excess weight following bariatric surgery," Journal of the American College of Nutrition, vol. 7, no. 2, pp. 147-153, 1988.

[14] P. Marceau, D. Kaufman, S. Biron et al., "Outcome of pregnancies after biliopancreatic diversion," Obesity Surgery, vol. 14, no. 3, pp. 318-324, 2004.

[15] A. Abodeely, G. D. Roye, D. T. Harrington, and W. G. Cioffi, "Pregnancy outcomes after bariatric surgery: maternal, fetal, and infant implications," Surgery for Obesity and Related Diseases, vol. 4, no. 3, pp. 464-471, 2008.

[16] J. R. Wax, M. G. Pinette, A. Cartin, and J. Blackstone, "Female reproductive issues following bariatric surgery," Obstetrical and Gynecological Survey, vol. 62, no. 9, pp. 595-604, 2007.

[17] W. L. Bennett, M. M. Gilson, R. Jamshidi et al., "Impact of bariatric surgery on hypertensive disorders in pregnancy: retrospective analysis of insurance claims data," British Medical Journal, vol. 340, p. c1662, 2010.

[18] A. Y. Weintraub, A. Levy, I. Levi, M. Mazor, A. Wiznitzer, and E. Sheiner, "Effect of bariatric surgery on pregnancy outcome," International Journal of Gynecology and Obstetrics, vol. 103, no. 3, pp. 246-251, 2008.

[19] A. C. Wittgrove, L. Jester, P. Wittgrove, and G. W. Clark, "Pregnancy following gastric bypass for morbid obesity," Obesity Surgery, vol. 8, no. 4, pp. 461-464, 1998.

[20] J. A. Patel, N. A. Patel, R. L. Thomas, J. K. Nelms, and J. J. Colella, "Pregnancy outcomes after laparoscopic Roux-en-Y gastric bypass," Surgery for Obesity and Related Diseases, vol. 4, no. 1, pp. 39-45, 2008.

[21] E. Sheiner, A. Levy, D. Silverberg et al., "Pregnancy after bariatric surgery is not associated with adverse perinatal outcome," American Journal of Obstetrics and Gynecology, vol. 190, no. 5, pp. 1335-1340, 2004. 
[22] H. M. Ehrenberg, B. M. Mercer, and P. M. Catalano, "The influence of obesity and diabetes on the prevalence of macrosomia," American Journal of Obstetrics and Gynecology, vol. 191, no. 3, pp. 964-968, 2004.

[23] G. Ducarme, A. Revaux, A. Rodrigues, F. Aissaoui, I. Pharisien, and M. Uzan, "Obstetric outcome following lapa-roscopic adjustable gastric banding," International Journal of Gynecology and Obstetrics, vol. 98, no. 3, pp. 244-247, 2007.

[24] D. S. Richards, D. K. Miller, and G. N. Goodman, "Pregnancy after gastric bypass for morbid obesity," Journal of Reproductive Medicine for the Obstetrician and Gynecologist, vol. 32, no. 3, pp. 172-176, 1987.

[25] J. B. Dixon, M. E. Dixon, and P. E. O’Brien, "Birth outcomes in obese women after laparoscopic adjustable gastric banding," Obstetrics and Gynecology, vol. 106, no. 5, pp. 965-972, 2005.

[26] T. Dao, J. Kuhn, D. Ehmer, T. Fisher, and T. McCarty, "Pregnancy outcomes after gastric-bypass surgery," American Journal of Surgery, vol. 192, no. 6, pp. 762-766, 2006.

[27] S. D. McDonald, Z. Han, S. Mulla, and J. Beyene, "Overweight and obesity in mothers and risk of preterm birth and low birth weight infants: systematic review and meta-analyses," British Medical Journal, vol. 341, p. c3428, 2010.

[28] B. Bilenka, I. Ben-Shlomo, C. Cozacov, C. H. Gold, and S. Zohar, "Fertility, miscarriage and pregnancy after vertical banded gastroplasty operation for morbid obesity," Acta Obstetricia et Gynecologica Scandinavica, vol. 74, no. 1, pp. 4244, 1995.

[29] P. Marceau, D. Kaufman, S. Biron et al., "Outcome of pregnancies after biliopancreatic diversion," Obesity Surgery, vol. 14, no. 3, pp. 318-324, 2004.

[30] D. Friedman, S. Cuneo, M. Valenzano et al., "Pregnancies in an 18-year follow-up after biliopancreatic diversion," Obesity Surgery, vol. 5, no. 3, pp. 308-313, 1995.

[31] C. Poitou Bernert, C. Ciangura, M. Coupaye, S. Czernichow, J. L. Bouillot, and A. Basdevant, "Nutritional deficiency after gastric bypass: diagnosis, prevention and treatment," Diabetes and Metabolism, vol. 33, no. 1, pp. 13-24, 2007.

[32] A. Eerdekens, A. Debeer, G. Van Hoey et al., "Maternal bariatric surgery: adverse outcomes in neonates," European Journal of Pediatrics, vol. 169, no. 2, pp. 191-196, 2010. 


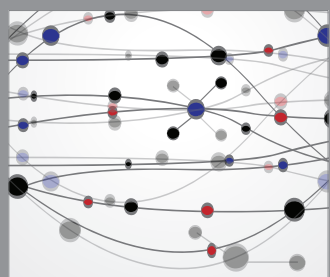

The Scientific World Journal
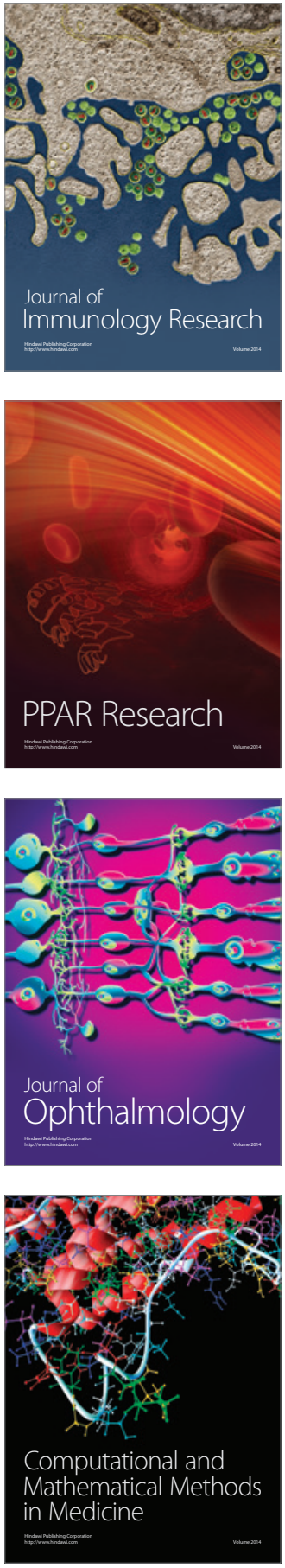

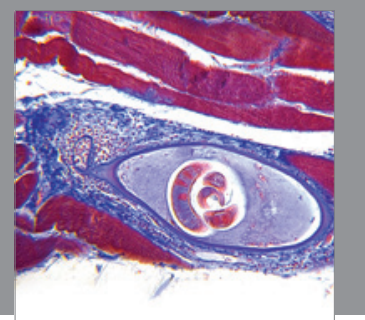

Gastroenterology

Research and Practice
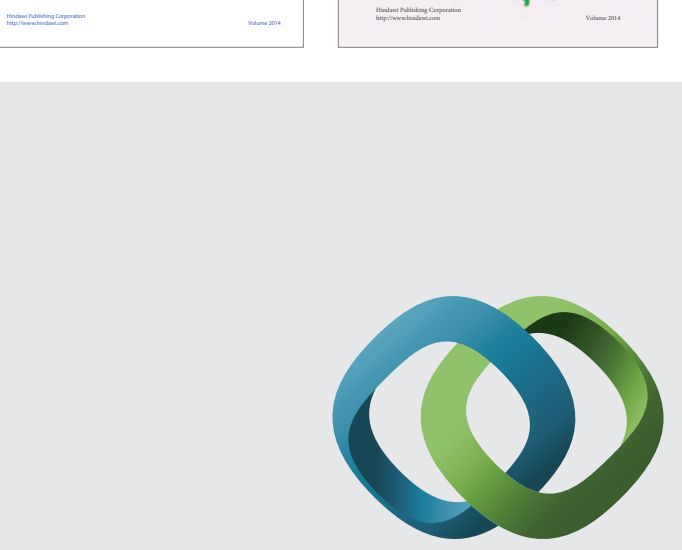

\section{Hindawi}

Submit your manuscripts at

http://www.hindawi.com
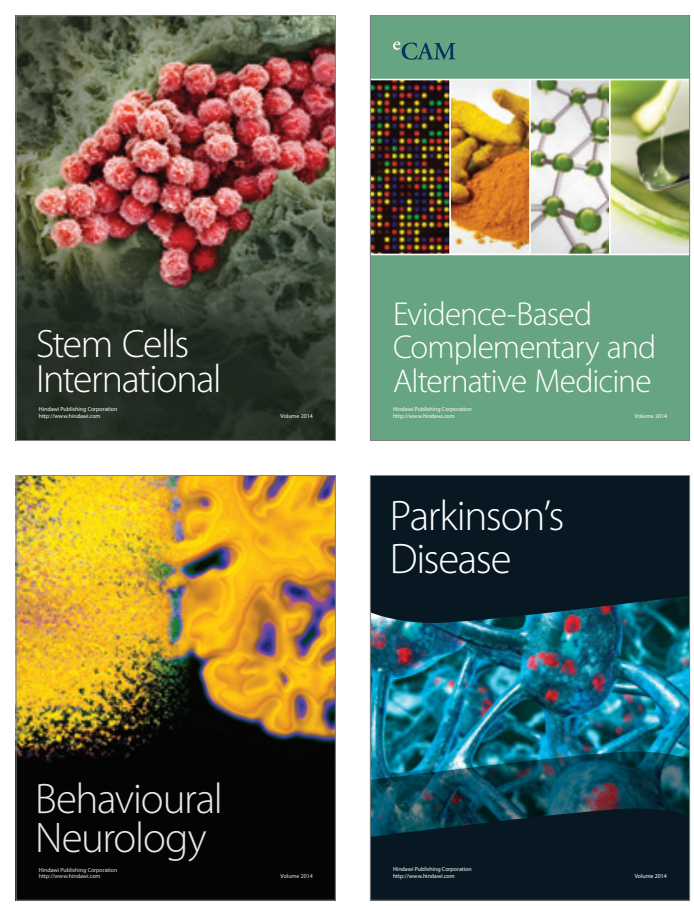

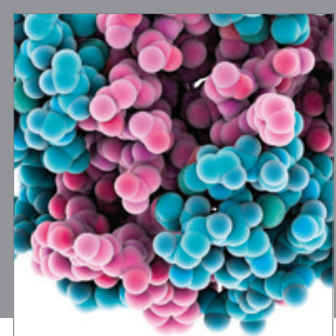

Journal of
Diabetes Research

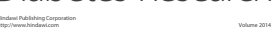

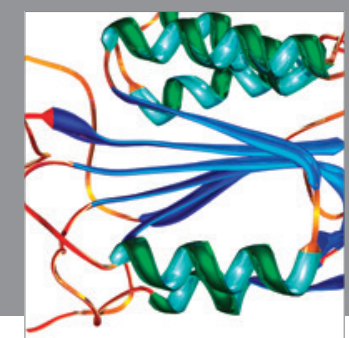

Disease Markers
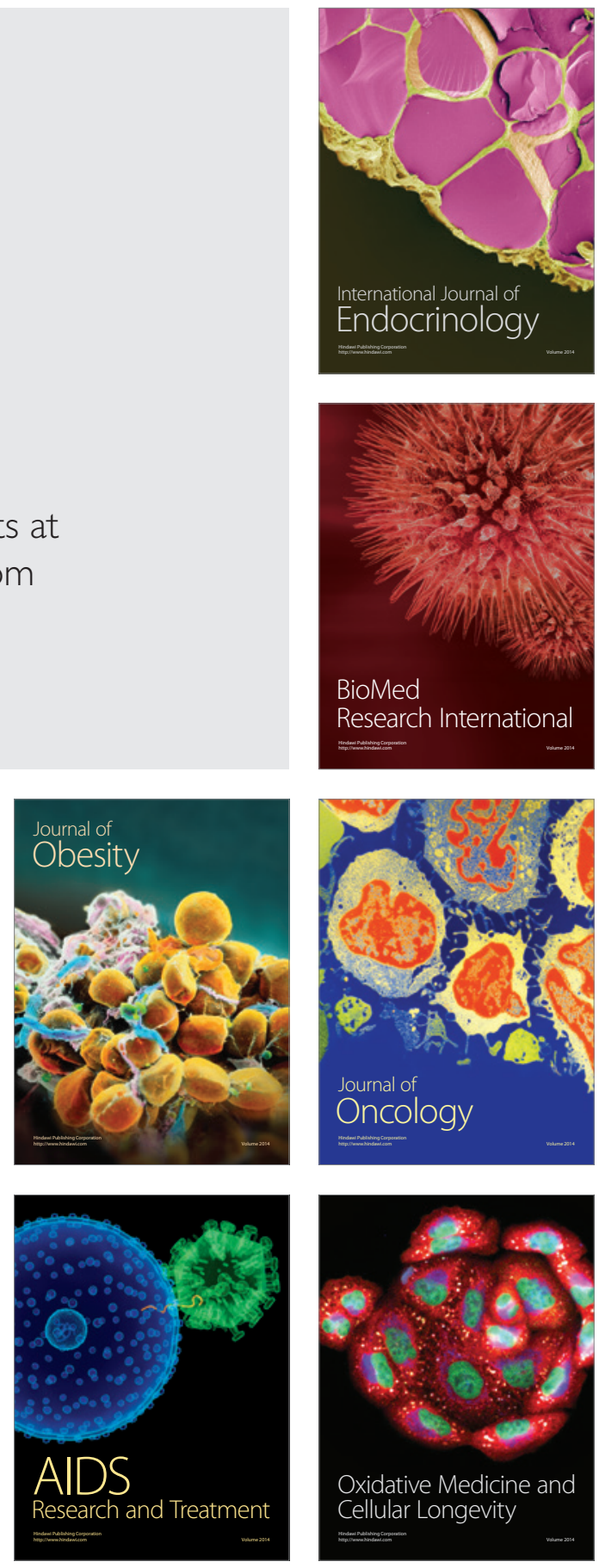